\title{
Integration of HIV/AIDS and noncommunicable diseases in developing countries: rationale, policies and models
}

\author{
Tilahun Nigatu Haregu*1, Geoffrey Setswe ${ }^{2}$, Julian Elliott ${ }^{3}$, Brian Oldenburg ${ }^{4}$ \\ ${ }^{1}$ African Population and Health Research Center, Nairobi, Kenya \\ ${ }^{2}$ Human Sciences Research Council, Pretoria, South Africa \\ ${ }^{3}$ Burnet institute, Melbourne, Australia \\ ${ }^{4}$ University of Melbourne, Melbourne, Australia
}

Received: June 26, 2015

DOI: $10.5430 /$ ijh.v1n1p21
Accepted: July 30, 2015

Online Published: August 7, 2015

\begin{abstract}
Background: HIV/AIDS and Noncommunicable disease (NCDs) are major public health problems in developing countries. An integrated approach of response to these problems is recommended. However, the evidence-base for such an approach is limited. The objective of this study was to consolidate evidence related to the rationale, the policy basis and models of HIV-NCD integration in developing countries.

Methods: Analysis of evidence related to the rationale for HIV-NCD integration, the policy basis for HIV-NCD integration, and models of HIV-NCD integration was conducted. As the evidence-base for HIV-NCD integration is very limited, a purposive and targeted search of the literature was used. Information was extracted using an abstraction tool. The extracted information was then aggregated and undergone qualitative synthesis under pre-defined themes.

Results: There is strong epidemiological, clinical and management related evidence relevant to the rationale for the integration of responses to HIV/AIDS and NCDs. Global declarations and strategies are in favour of integrated response to HIV/AIDS and NCDs. Types of models of HIV-NCD integration that were "tested" in the context of developing countries vary by contexts, disease combinations, types of services, and number of disease conditions involved. Despite these variations, all the models for which outcomes are available indicated that the integrated approach was feasible, effective, efficient and acceptable.

Conclusions: Evidence about rationale, policy bases and existing models of HIV-NCD integration from developing countries strongly favours integrated response. However, this is limited in breadth and depth. Therefore, more empirical evidence is needed to better inform decisions related to of HIV-NCD integration.
\end{abstract}

Key Words: Integration, HIV/AIDS, Noncommunicable disease, Developing countries

\section{BACKGROUND}

Communicable diseases, maternal and child health problems, and injuries are still profoundly impacting on the health and economic development of those countries. Globally, an es- timated 35 million people were living with HIV (PLHIV) in 2014. Since the beginning of the epidemic, almost 70 million people have been infected with the HIV virus and about 35 million people have died of AIDS. Sub-Saharan

\footnotetext{
* Correspondence: Tilahun Nigatu Haregu; Email: tilahunigatu@gmail.com; Address: African Population and Health Research Center, P.O.Box 10787-00100, Nairobi, Kenya.
} 
Africa remains to be the most severely affected, with nearly $5 \%$ of adults living with HIV and accounting for $69 \%$ of the PLHIV worldwide. ${ }^{[1,2]}$

Noncommunicable diseases (NCDs) kill more than 36 million people each year. Majority of these deaths occur in low and middle income countries. More than $25 \%$ of all NCD deaths occur before age of 60 ; and $90 \%$ of these premature NCD deaths occur in low and middle income countries. The four common NCDs (cardiovascular diseases, Cancers, Diabetes and Chronic obstructive pulmonary disease) which share four common risk factors account for around $80 \%$ of all NCD deaths. In Sub-Saharan Africa, where average age at death has risen by less than 10 years, Noncommunicable diseases account less than $50 \%$ of all health years lost. ${ }^{[3]}$

A lot has been invested to curb the tides of HIV/AIDS in developing countries. However, some challenges still remain. ${ }^{[2]}$ While the agenda of communicable diseases is still unfinished, these countries need to prioritize the fight against NCDs. Along with the emergence of NCDs in developing countries, there is a consistent conversation about integrated response, a strategy through which key actors and their actions are well synchronized to address health needs. However, the evidence-base to support the argumentation is limited. A recent study about evidence for NCDs indicated that the overwhelming body of evidence for NCDs is from developed countries. ${ }^{4]}$ Integration with HIV could pave the way for NCD response to learn from HIV responses. In this regard, there are some disease and service specific attempts to integrate NCD services in to HIV/AIDS programs in developing countries. These efforts used different models in different settings for different combinations of disease entities. With these premises, this study was designed to consolidate evidence under three essential themes of HIV-NCD integration: rationale, policy bases, and models.

\section{Methods}

An interpretive qualitative synthesis of evidence related to HIV-NCD integration was conducted. For this study, NCDs were limited in to the four common NCDs. As the evidencebase for HIV-NCD integration is very limited, the authors conducted a purposive and targeted search of the literature using search terms relevant to HIV/AIDS, NCDs (including the four specific NCDs) and integration. Information sources including PubMed and general search engine as well as the website of major global organizations working on HIV/AIDS and Noncommunicable diseases including World Health Organization (WHO), UNAIDS, and the NCD alliance were explored for relevant documents. The most recent national HIV/AIDS and NCD strategic plans of four purposively selected countries were also examined for policy statements relevant to integration. In all cases, the inclusion of references was restricted to those published between 2006 and 2014.

Relevant information was retrieved from the selected sources. The extracted information was then aggregated and undergone qualitative synthesis under pre-defined themes. Resulting evidence was categorized based on the basic foundations of argumentation for rationale, major policy anchors of the HIV/AIDS and NCD response, and types of models of integration. Information in each category was presented using narrative summaries. Finally, evidence from the three dimensions was consolidated using interpretive synthesis.

\section{RESUltS}

\subsection{Rationale}

Analysis of the evidence about the rationale for the integration of responses to HIV/AIDS and NCDs revealed five major reasons relevant to HIV-NCD integration.

\subsubsection{Similarities}

Chronicity (gradual development and slow progression) is a key characteristic of both HIV/AIDS and the common NCDs. In the era of anti-retroviral treatment, HIV has turned out to be a chronic disease of infectious origin. ${ }^{[5]}$ Therefore, a chronic disease management approach would be applicable for the prevention and control of both HIV/AIDS and NCDs.

\subsubsection{Convergence}

Epidemiological evidence suggests that developing countries bear the highest burden of both HIV/AIDS and NCDs. This is due to high population size, increasing life expectancy and rapidly changing lifestyles that resulted in increase of NCD in countries which have an already high HIV prevalence. As a result, the main focus of global HIV and NCD prevention and control efforts are in developing countries. Overlapping problems require coordinated responses. In this regard, HIV-NCD integration is an important policy issue.

\subsubsection{Linkage}

HIV and NCDs are inter-connected both in terms of risk of development of disease, and severity of progression of disease: HIV increases the risk of developing NCDs, and NCDs aggravate the severity of HIV disease. ${ }^{[6]}$ Therefore, the development and implementation of intervention packages need to consider the linkages between HIV/AIDS and NCDs. Integrated response required well-aligned intervention packages. Therefore, connection between HIV and NCDs could be considered as an important driver of integrated response.

\subsubsection{Co-occurrence}

Due to the connection between HIV/AIDS and NCDs, cooccurrence of the diseases in an individual, comorbidity, is 
common. Evidence about levels of comorbidity is essential for planning and management of multi-morbid cases. Because of the effects of HIV disease itself, HIV treatment effects and increasing age, the HIV positive population is at a higher risk of NCDs than the general population. ${ }^{[7]} \mathrm{HIV}$ patients having NCDs need comprehensive services that have continuity. This goal is achieved through integration that maximizes efficiency and synergy.

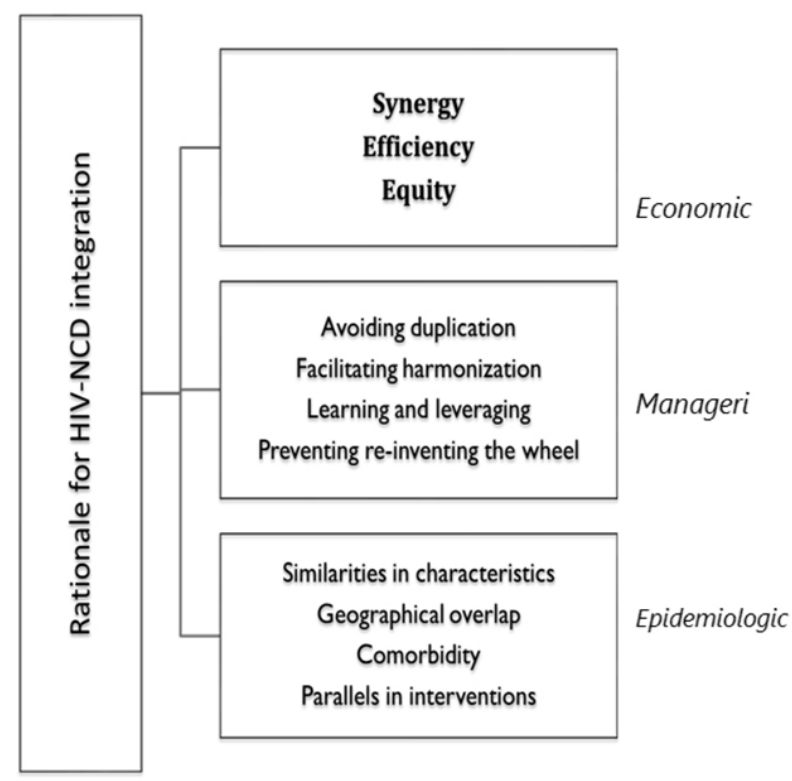

Figure 1. Three levels of rationale for HIV-NCD integration It shows the levels of rationale for integration of the responses to HIV/AIDS and NCDs. The various rationale found in the analysis are categorized in to epidemiological, managerial and economic rationales.

\subsubsection{Commonalities}

The responses to HIV/AIDS and NCDs have many commonalities. Long-term treatment, regular monitoring, adherence to treatment and lifestyle modifications are the essential elements of management of both disease conditions. Multisectoral approaches, whole-of-government involvement and whole-of-society efforts are critical to the responses to both HIV/AIDS and NCDs. Many of the health system challenges are also common to both HIV and NCDs. ${ }^{[8]}$

\subsection{Policy bases}

Exploration of the policy bases for integration of the responses to HIV/AIDS and NCDs indicated that almost all existing global Health, HIV/AIDS and NCD policy statements and strategies favour the integration of responses to HIV/AIDS and NCDs.

\subsubsection{HIV/AIDS declaration 2011}

This declaration emphasizes on the achievement of Millennium Development Goals (MDGs). It recognises the importance of integrating HIV/AIDS prevention, treatment, and care and support services with efforts that are important to achieve MDGs. The declaration also emphasizes on the integration of high impact HIV interventions in to the activities of different sectors. Integrating HIV response in to primary healthcare, including Maternal and Child Healthcare, is also highlighted. Besides, the integration of Tuberculosis, hepatitis, and food and nutrition support interventions in to HIV response in underscored. ${ }^{[9]}$ HIV-NCD integration is implied throughout this declaration.

\subsubsection{Global health sector strategy on HIV/AIDS 2011- 2015}

One of the main themes in this strategy is about attaining better integration of HIV programs with other health programs (including NCDs). Providing comprehensive and integrated services for key populations is among the core elements of the strategy. Linking programs and integrating HIV in to other health services is highlighted. The strategy also states that HIV programs have assisted in integrating chronic disease management in resource limited settings. As per the strategy, promoting the linkage between HIV and other priority health programs (including NCDs) is among the WHO contributions. Among the recommended country actions is strengthening the management of both HIV and Noncommunicable and chronic diseases. Maximizing synergy across other programs (including NCDs) is also among WHO's optimizing activities. ${ }^{[10]}$

\subsubsection{UNAIDS strategy 2011-2015}

This strategy supports the strengthening of Health systems to deliver decentralized and integrated services. TB/HIV integration and leveraging services for the elimination of vertical transmission of HIV at antenatal, delivery and child health services are underlined. This strategy also articulates the integration of HIV response with other health and development efforts. To address women's right in the context of HIV, integration of HIV with sexual and reproductive health programs is among the strategic issues. ${ }^{[11]}$ This strategy doesn't specifically call for HIV-NCD integration. However, there is a report about how to leverage HIV experience for NCDs prepared by UNAIDS.

\subsubsection{UN declaration on NCDs 2011}

Article 27 of United Nations General Assembly Political Declaration on the Prevention and Control of NCDs unequivocally states "Note with concern the possible linkages between NCDs and some communicable diseases, such as HIV/AIDS, and call to integrate, as appropriate, responses for HIV/AIDS 
and NCDs and, in this regard, for attention to be given to people living with HIV/AIDS, especially in countries with a high prevalence of HIV/AIDS and in accordance with national priorities." Additional articles in the same declaration re-iterate this call by recommending learning from experiences in the field of HIV/AIDS. ${ }^{[12]}$

\subsubsection{Global action plan on NCDs 2013-2020}

Based on this action plan, one of the priority actions for member states is "to review existing HIV/AIDS programs for opportunities to integrate in to them service delivery for the prevention and control of NCDs". The NCD action plan also reaffirms "integrating NCD programmes or palliative care with HIV care programmes would bring mutual benefits since both cater to long-term care and support as a part of the programme and also because NCDs can be a side-effect of long-term treatment of HIV infection and AIDS ${ }^{[13]}$ ".

\subsubsection{National HIV/AIDS policy frameworks}

HIV/AIDS strategic frameworks of all four countries give due consideration for the integration of different components of HIV/AIDS response. HIV/AIDS strategy framework of Ethiopia emphasizes of the integration of HIV/AIDS interventions with other (Health) programs. ${ }^{[14]}$ The national HIV Strategic plan of Sri Lanka, which is developed more recently than the others, also underlines the integration of HIV/AIDS programs in to the activities of different sectors. ${ }^{[15]}$ In Malaysia, one of the focus of the HIV/AIDS strategy framework is "Strengthening priority health services through increased integration of HIV service delivery ${ }^{[16] "}$ ". The National HIV/AIDS Strategy framework of South Africa go further and calls for integration of the screening of common NCDs and their risk factors with outreach HIV counselling and testing programs. ${ }^{[17]}$

\subsubsection{National NCD policy frameworks}

National NCD strategy frameworks on the other hand underscore integrated NCD response and integration of NCD response in to health and other related systems. For instance, one of the objectives of the National NCD strategic plan of Malaysia is to integrate prevention and control of such diseases into policies across all government departments. This NCD strategic plan was meant to integrate the various frameworks, strategies and action plans addressing specific risk factors and particular diseases into a holistic and definitive approach to NCD prevention and control. ${ }^{[18]}$ Similarly, the National NCD strategic framework of Sri Lanka aims to integrate prevention and control of NCDs into policies across all government ministries and private sector organisations. ${ }^{[19]}$

The NCD strategic framework of Ethiopia considers NCD response as an integral part of primary health care and Health Extension Program. It aims to establish a program for surveil- lance of chronic disease risk factors, as an integral part of the communicable disease surveillance and response system. ${ }^{[20]}$ Based on the principle of Integrated Chronic Disease Management Model, the NCD strategic framework of South Africa aims to integrate NCDs in to the re-engineering of primary Health care in that country. This framework explicitly states the integration of HIV testing and NCD screening. [21]

In general, three forms of integration are addressed by the above mentioned policy frameworks. These are integration of HIV/AIDS and NCD responses into the health system, integration within HIV/AIDS or NCD response (intra-), and integration between HIV/AIDS and NCD responses (inter-). The degree of emphasis given for these forms of integration is indicated in Table 1.

Table 1. Degree of policy support given to the three forms of integration

\begin{tabular}{lllll}
\hline \multirow{6}{*}{$\begin{array}{l}\text { Policy } \\
\text { frameworks }\end{array}$} & $\begin{array}{l}\text { "In to" } \\
\text { health } \\
\text { system }\end{array}$ & $\begin{array}{l}\text { "Within" } \\
\text { HIV or }\end{array}$ & $\begin{array}{l}\text { "Between" } \\
\text { HIV and } \\
\text { Global }\end{array}$ & NCD \\
\hline \multirow{6}{*}{ National } & Health & +++ & + & + \\
& HIV/AIDS & ++ & +++ & + \\
& NCD & +++ & +++ & +++ \\
& Health & +++ & + & + \\
& HIV/AIDS & ++ & +++ & + \\
& NCD & +++ & ++ & $+/++$ \\
\hline
\end{tabular}

Note. +: Moderate support; ++: Strong support; +++: Very strong support

\subsection{Models}

This analysis has identified a few feasibility studies from developing countries. These studies are limited to specific settings and contexts within those countries. The categorization of the models in to the four overlapping dimensions (see Figure 2) is based on analysis of the main functions considered in the integration.

\subsubsection{Cervical cancer screening and HIV}

There are many small scale studies reporting on the integration of Cervical Cancer screening in to HIV programs in many countries in Africa. ${ }^{[22]}$ These initiatives involve the use of Visual inspection with Acetic acid (VIA) as a screening method. The studies were conducted between 2007 and 2012. In all the studies, integration of cervical cancer screening in to HIV services was reported to be feasible, effective, efficient and acceptable. The challenges faced were related to capacity and loss to follow up.

\subsubsection{Chronic disease clinic}

This model used full integration of HIV and NCD services in to a chronic disease clinic in Cambodia. ${ }^{[23]} \mathrm{HIV}, \mathrm{Hy}-$ pertension and Diabetes care and treatment services were integrated. The newly established chronic disease clinics 
were at provincial hospitals and were supported by external funding but gradually taken by Ministry of Health. The service delivery in the clinics had strong emphasis on outpatient consultations. After implementation for three years, it was proved that integration of HIV and NCDs is feasible, effective, efficient and acceptable in that context.

\subsubsection{Adapting HIV strategies, systems and tools for dia- betes}

This model was about leveraging HIV programs to support diabetes services. It was implemented in Ethiopia and Swaziland. ${ }^{[24]}$ A project was implemented to evaluate the feasibility and effectiveness of adapting HIV program-related tools and systems for patients with Diabetes. The intervention package included strategies, systems and tools adapted from HIV clinic and applied to diabetes services to outpatient department. The outcomes have illustrated that "countries which have successfully scaled up HIV services have already learned profound lessons about the delivery of chronic care. Using these locally owned and contextually appropriate resources may be an efficient and effective way to 'jumpstart' NCD programs and to strengthen health systems to support longitudinal services for all".

\subsubsection{Rapid multi-disease testing screening}

This model involved leveraging rapid community-based HIV testing campaigns for NCDs in rural Uganda. The study tested the feasibility and diagnostic yield of integrating NCD and communicable diseases in to rapid HIV testing and referral campaign. The multi-disease campaign included provision of diagnostic, preventive, therapeutic and referral services for HIV, Malaria, Tuberculosis, Hypertension and Diabetes at a point-of-care. The campaign demonstrated the feasibility of integrating hypertension, diabetes and communicable diseases into HIV/AIDS initiatives. ${ }^{[25]}$

\subsubsection{Integrating cardiovascular disease screening in to HIV services}

This model is a pilot project in Kenya. The pilot program offers Cardiovascular disease (CVD) screening for people attending healthcare facilities for HIV prevention and treatment. ${ }^{[26]}$ HIV Service providers were trained to screen CVD risk factors using adapted behaviour materials and job aids. Counselling and referral linkage are offered for clients in need. Ministry of Health and National AIDS and STI control program were engaged. It is reported that the recent strategic plan now calls for integration of HIV and CVD screening because of the inspiration of this project.

\subsubsection{Integrated chronic disease management model}

South African Department of Health introduced a Health System Framework to strengthen the response to NCDs: the Integrated Chronic Disease Management Model (ICDM).
The model is for chronic diseases, both NCDs and communicable disease, notability HIV and Tuberculosis. ${ }^{[21]}$ The model is based on re-engineering of the primary healthcare process. Results are yet to be reported.

\subsubsection{Integrating NCD risk factor and HIV/AIDS inter- ventions}

The integration of substance abuse treatment - Smoking cessation and alcohol abuse prevention - is studied in the developed world. A study conducted in Botswana recommended the integration of alcohol abuse and HIV prevention efforts. ${ }^{[27]}$ The importance of smoking cessation interventions in HIV care are underscored by a study from Nepal. ${ }^{[28]}$ Evidence on the integration of physical activity and Nutritional interventions among PLHIV in developing countries is limited. These models are centred on four focal points: Problem, People, Process, Patient, and patient (see Figure 2). These points are not mutually exclusive.

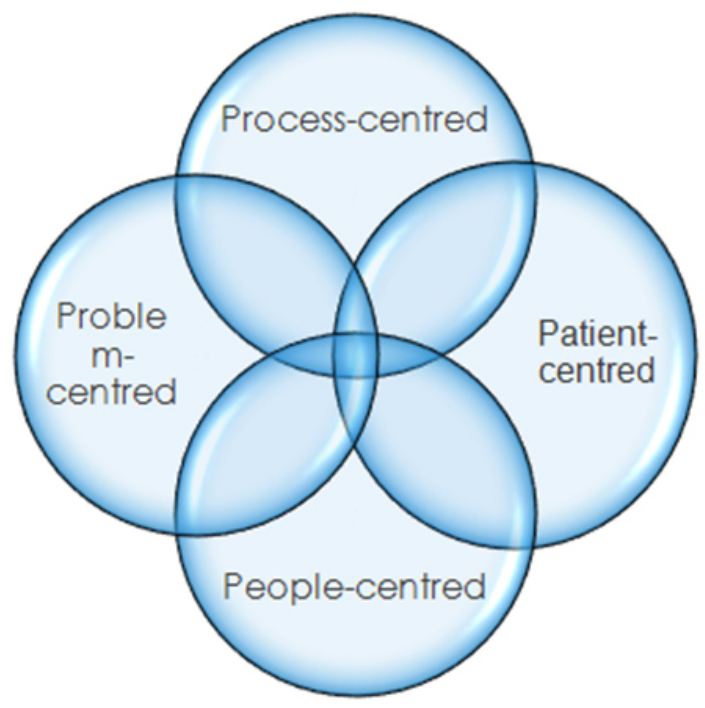

Figure 2. Categories of HIV-NCD service integration models

\section{Discussion}

There is strong epidemiological, clinical and management related evidence base on the illustrating the rationale for the integration of health system responses to HIV/AIDS and NCDs in developing countries. However, the relevance of this evidence may vary based on the "balance" between the burden of HIV/AIDS and NCDs in different countries. ${ }^{\text {[29] }}$ The evidence strongly supports integration for high HIV and high NCD burden contexts where the "doses" of the responses are proportional. ${ }^{[5]}$

Global HIV/AIDS declarations and strategies are in favour of integrated health system response in general though these have not been specifically articulated in terms of HIV-NCD 
integration. Additional position statements, however, explicitly state HIV-NCD integration as a preferred approach for developing countries. ${ }^{[8,30]}$ On the other hand, the NCD declaration and action plan unequivocally adopted integrated approach, including that with HIV, as a primarily preferred strategy. The priorities of National strategies seem to vary based on epidemiological profiles. The actual implementation of these policies and strategies should also focus on integrated approaches. However, the "how" and the "what" components is are not adequately addressed or left for specific levels of the health system.

Models are limited to Health Services integration, mostly for HIV and one NCD. The types of models of HIV-NCD integration that were "tested" in the context of developing country vary by country contexts, disease combinations, types of services, and number of disease conditions involved. Despite these variations, all the models for which outcomes are available indicated that the integrated approach was feasible, effective, efficient and acceptable. In this regard, developing and testing additional models of integration that can address broader contexts is needed.

There exists a strong rationale and a well-founded policy basis for integration of the responses to HIV/AIDS and NCDs in developing countries. The presence of various models of integration reflects the differences in the health system environment and disease epidemiology among different countries. Moreover, integration of different services at a single site can be expensive. Therefore, context specific models which promote stronger interactions among HIV/AIDS and NCD actors and sectors are needed. Such models would avoid missed opportunities as well as unanticipated problems that could arise from integration efforts.

There is limited evidence about HIV-NCD integration both in terms of scope and generalizability of the evidence. Existing evidence is also limited to small scale feasibility studies in largely different contexts. There were no large scales or randomized and/or controlled evaluations. As a result, for some of the models, data on effectiveness outcomes are yet to be available in the near future.

\section{Conclusions}

This synthesis has consolidated the evidence-base on the rationale, policy bases and existing models of HIV-NCD integration in the context of developing countries. The resulting evidence strongly favours integrated response. However, the overall evidence in this arena is limited and contextspecific evidence is lacking. Therefore, more research-based evidence is needed to better inform decisions related to HIVNCD integration. More evidence is needed to advocate for the integration and future efforts need to focus on advocating for policy/operational research on HIV-NCD integration.

\section{CONFlicts OF INTEREST Disclosure}

The authors declare that there are no competing interests associated with this study.

\section{REFERENCES}

[1] Murray CJ, Ortblad KF, Guinovart C, et al. Global, regional, and national incidence and mortality for HIV, tuberculosis, and malaria during 1990-2013: a systematic analysis for the Global Burden of Disease Study 2013. Lancet. 2014; 384(9947): 1005-70. http: //dx.doi.org/10.1016/S0140-6736(14)60844-8

[2] UNAIDS. Global AIDS Report: UNAIDS report on the global AIDS epidemic 2013. UNAIDS: 20 Avenue Appia, CH-1211 Geneva 27, Switzerland; 2013

[3] Institute for Health Metrics and Evaluation. The Global Burden of Disease: Generating Evidence, Guiding Policy. Seatle, WA: IHME; 2013.

[4] Heneghan C, Blacklock C, Perera R, et al. Evidence for noncommunicable diseases: analysis of Cochrane reviews and randomised trials by World Bank classification. BMJ Open. 2013; 3(7): 2013-003298. PMid: 23833146. http://dx.doi.org/10.1136 /bmjopen-2013-003298

[5] Nigatu T. Integration of HIV and noncommunicable diseases in health care delivery in low- and middle-income countries. Prev Chronic Dis 2012; 9: 3. http://dx.doi.org/10.5888/pcd9.110331

[6] Nigatu T, Setswe G, Elliott J, et al. Developing a Public Health Framework for the Epidemiological Linkages between HIV/AIDS and NCDs: A Thematic Research Synthesis. International Journal of Prevention and Treatment. 2012; 1(4): 53-60. http://dx.doi.org /10.5923/j.ijpt. 20120104.01

[7] Nigatu T, Oldenburg B, Elliot J, et al. The incidence of cardiovascular disease, cancer and type 2 diabetes comorbidities in HIV infection: A systematic review. Journal of Nursing Education and Practice. 2013; 3(7): 58. http://dx .doi.org/10.5430/jnep.v3n7p58

[8] Haregu TN, Setswe G, Elliott J, et al. National Responses to HIV/AIDS and Non-Communicable Diseases in Developing Countries: Analysis of Strategic Parallels and Differences. Journal of public health research. 2014; 3(1): 99. PMid: 25170505. http: //dx.doi.org/10.4081/jphr.2014.99

[9] Assembly UG. Political Declaration on HIV and AIDS: Intensifying Our Efforts to Eliminate HIV and AIDS. 2011.

[10] World Health Organization. Global health sector strategy on HIV/AIDS 2011-2015.: WHO Press, World Health Organization, 20 Avenue Appia, 1211 Geneva 27, Switzerland; 2011.

[11] UNAIDS. Getting Zero: UNAIDS 2011-2015 Strategy. UNAIDS: 20 Avenue Appia, CH-1211 Geneva 27, Switzerland; 2010.

[12] United Nations General Assembly. Political declaration of the Highlevel Meeting of the General Assembly on the Prevention and Control of Non-communicable Diseases. 2011. 
[13] World Health Assembly. Global Action Plan for The Prevention and Condtrol of Noncommunicable diseases 2013-2020. 2013.

[14] Federal HIV/AIDS Prevention and Control office. Strategic Plan II for intensifying multisectoral HIV and AIDS response in Ethiopia 2010/11-2014/15. Addis Ababa, Ethiopia: Federal HIV/AIDS prevention and control office; 2010.

[15] National STD/AIDS Control Programme. National HIV Strategic plan Sri Lanka 2013-2017. Sri Lanka: Ministry of Health; 2013.

[16] Health Mo. National Strategy on HIV and AIDS 2011-2015. Malaysia: Ministry of Health; 2011. PMid: 21654546.

[17] South African National AIDS Council. National Strategic plan for HIV, STIs and TB 2012-2016. South African National AIDS council; 2012.

[18] Ministry of health. National Strategic Plan for Noncommunicable diseases: Medium-term strategic plan to further strengthen cardiovascular disease and diabetes prevention and control program in Malaysia (2010-2014). In: Noncommunicable disease section Dcd, editor. Malaysia 2010.

[19] Ministry of Healthcare and Nutrition. The National Policy and Strategic framework for prevention and control of chronic Noncommunicable diseases. Sri Lanka: Ministry of Healthcare and Nutrition; 2009.

[20] Federal Ministry of Health. Prevention and control of chronic Noncommunicable diseases: A strategic framework. Addis Ababa, Ethiopia: Ministry of Health; 2010.

[21] Health Do. South African Strategic Plan for the Prevention and Control of Non-Communicable Diseases 2013-17. South Africa. 2013.

[22] Plotkin M, Besana GV, Yuma S, et al. Integrating HIV testing into cervical cancer screening in Tanzania: an analysis of routine service delivery statistics. BMC women's health. 2014; 14: 120. PMid: 25271025. http://dx.doi.org/10.1186/1472-6874-14-120

[23] Janssens B, Van Damme W, Raleigh B, et al. Offering integrated care for HIV/AIDS, diabetes and hypertension within chronic disease clinics in Cambodia. Bull World Health Organ. 2007; 85(11): 880-5. PMid: 18038079.

[24] Rabkin M, Melaku Z, Bruce K, et al. Strengthening Health Systems for Chronic Care: Leveraging HIV Programs to Support Diabetes Services in Ethiopia and Swaziland. Journal of Tropical Medicine. 2012; 2012: 6. PMid: 23056058. http://dx.doi.org/10.1155 /2012/137460

[25] Chamie G, Kwarisiima D, Clark TD, et al. Leveraging rapid community-based HIV testing campaigns for non-communicable diseases in rural Uganda. PLoS One. 2012; 7(8): 20. PMid: 22916256 http://dx.doi.org/10.1371/journal . pone. 0043400

[26] Center for Health Market Innovations. FHI 360 Cardiovascular Disease and HIV Integration 2010 [Nov 4, 2013]. Available from: http://healthmarketinnovations.org/program/fh i-360-cardiovascular-disease-and-hiv-integration

[27] Weiser SD, Leiter K, Heisler M, et al. A Population-Based Study on Alcohol and High-Risk Sexual Behaviors in Botswana. PLoS Med. 2006; 3(10): e392. PMid: 17032060. http://dx.doi.org/10.13 71/journal.pmed.0030392

[28] Amiya RM, Poudel KC, Poudel-Tandukar K, et al. Physicians are a key to encouraging cessation of smoking among people living with HIV/AIDS: a cross-sectional study in the Kathmandu Valley, Nepal. BMC Public Health. 2011; 11(677): 1471-2458. http://dx.doi.org/10.1186/1471-2458-11-677

[29] Marquez PV, Suhrcke M. Combating non-communicable diseases. BMJ. 2005; 331(7510): 174. PMid: 16037424. http://dx.doi.o $\mathrm{rg} / 10.1136 / \mathrm{bmj} .331 .7510 .174$

[30] Haregu TN, Oldenburg B, Elliott J, et al. Global Responses to HIV/AIDS and Noncommunicable Diseases: Analysis of Similarities and Differences. British Journal of Medicine \& Medical Research. 2013; 3(2): 442-65. PMid: 23277192. http://dx.doi.org/10. 9734/BJMMR/2013/2835 